\section{NEWS IN BRIEF \\ The University of Buckingham and the Leicester Dental Teaching Academy (LDTA) have reached an amicable agreement to end their joint venture to establish a dental school in Leicester to complement a new medi- cal school. A joint venture agreement was signed in April 2012, but delays to the anticipated start date for the medical school and the large amount of resource required for this project alone has left the university with no choice but to delay the dental school until the medical school is estab- lished. As the university is unable to commit to a start date, this has made it impossible for the LDTA to complete contracts for the provision and con- struction of facilities.}

The BDA has produced a series of short podcasts called 'In 10'. The podcasts combine a news angle with some of the advice and services the BDA offers to members and feature interviews with BDA experts and officers. The hot topics covered so far include tooth whitening, the $\mathrm{COC}$, the NHS pilots and most recently, dental care professionals. Members can access the podcasts at www.bda. org/news-centre/podcasts/in-ten/.

National Smile Month is due to take place 19 May-19 June 2014. In 2013, around 3,000 organisations supported the campaign and reached over 50 million people with a positive message to improve oral health. Organisers, the British Dental Health Foundation, are launching a new-look website for 2014. To register your interest in taking part and to receive your free Smileys, visit www. smilemonth.org.

Dog-lover Julie Cross is asking for $B D J$ readers to donate absolutely any medical/dental equipment, tools, medication or dressings they can spare to improve the lives of stray dogs. Registered charity Love UnderDogs work to improve the lives of stray dogs in Romania and rehome some of the most unwanted animals in the UK, such as those that are old, disabled, too big, the wrong colour or 'not pretty enough'. Discontinued and out of date products are welcome. www.loveunderdogs.org

\title{
CALCULUS IS A LONG-TERM RESERVOIR OF DISEASE
}

$\mathrm{T}$ he first detailed analysis of ancient oral microbiome ecology and function has revealed that despite significant changes in diet and lifestyle over the last 1,000 years, the oral cavity has been a long-term host for opportunistic pathogens that initiate oral and respiratory diseases, as well as bacteria associated with the development of heart disease and other systemic diseases.

The study published in Nature Genetics analysed four adult human skeletons from the medieval monastic site of Dalheim, Germany (c. 950-1200 AD), which presented evidence of mild to severe periodontal diseases, as well as the dental calculus of nine twenty-first century individuals with known dental histories. Previously, five common oral bacteria have been identified in historic dental calculus, but by applying shotgun DNA sequencing the researchers were able to distinguish 40 putative opportunistic pathogens, finding the calculus strongly dominated by bacterial DNA.

DNA sequences also revealed antibiotic resistance genes, suggesting that even before therapeutic antibiotics were used by humans, the oral cavity functioned as a source of low-level antibiotic resistance. Forty-three human proteins were identified in the calculus, 25 of which are involved in the innate immune system, driving inflammation and host defence processes. The wealth of inflammatory proteins supports the evidence of attachment loss and active periodontal disease.

As well as the researchers providing direct insight into common diseases of the human evolutionary past, their study also used plant and animal DNA sequences recovered from the ancient calculus to provide a high-resolution taxonomic characterisation of their diet. Animal tissue, plant bast fibres and starch granules from cereals were among their findings. These results have significant implications for archaeological studies but also future medical and microbiome research. Warinner et al. conclude that 'dental calculus is a robust, long-term biomolecular reservoir of ancient disease and dietary information'.

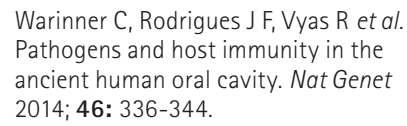
Pathogens and host immunity in the ancient human oral cavity. Nat Genet 2014; 46: 336-344.

BY LAURA PACEY

\section{CHILDREN SUSCEPTIBLE TO NEFARIOUS PARTNERSHIPS}

Early childhood caries may result from a nefarious partnership between a bacterium and a fungus, according to a new paper published in Infection and Immunity. ${ }^{1}$

Researchers at the University of Pennsylvania showed that infection by Streptococcus mutans and Candida albicans together doubled the number of cavities and boosted their severity several-fold in rats.

S. mutans has long been assumed to be the sole microbial culprit in the highly aggressive and painful form of tooth decay that frequently occurs in preschool children, especially from backgrounds of poverty. However, the researchers noticed that $C$. albicans was almost always present in plaque from cases of early childhood caries. S. mutans sticks to the teeth, whereas $C$. albicans sticks mainly to cheek and tongue but has rarely been seen in plaque until now.

The investigators discovered that the 'exoenzyme' which $S$. mutans uses to react with sugar, also enables $C$. albicans to produce a glue-like polymer in the presence of sugar, allowing it to adhere to teeth, and to bind $S$. mutans, two abilities it otherwise lacks. Under these circumstances, the fungus now contributes the bulk of the plaque.

The authors' research supports their hypothesis that early childhood caries in toddlers results from infection by both organisms, with frequent exposure to sucrose.

\section{Falsetta $M$ L, Klein M I, Colonne P M et al. Symbiotic relationships between Streptococcus mutans and Candida albicans synergizes the virulence of plaque- biofilms in vivo. Infect Immun 2014; DOI: 10.1128/IAI.00087-14}

